\title{
HIGH-RESOLUTION CO (1-0) OBSERVATIONS OF THE RINGED GALAXY NGC 4736
}

\author{
TONY WONG, TAMARA HELFER AND LEO BLITZ \\ University of California at Berkeley \\ Berkeley, CA 94720 U.S.A. \\ e-mail:twong@astro.berkeley.edu
}

\section{Introduction}

It is well known that the radial distributions of atomic and molecular gas differ markedly in spiral galaxies, including our own (e.g. Burton \& Gordon 1978 , Sofue et al. 1995). We have recently begun a program to obtain multifrequency observations of several nearby galaxies in order to determine whether $\mathrm{HI}$ is needed to replenish the $\mathrm{H}_{2}$ consumed by star formation and, if so, how this might be accomplished. Here we present initial results on the nearby RSab galaxy NGC 4736, known for its bright ring of H II regions and recently shown to have a central stellar bar (Möllenhoff et al. 1995). We have mapped the distribution of $\mathrm{CO}$ (1-0) emission within $r=1^{\prime}(1.8 \mathrm{kpc}$ at $D=6.2 \mathrm{Mpc}$ ) with the BIMA interferometer at $\sim 5^{\prime \prime}$ resolution, and added data from the NRAO 12-m telescope to recover zero-spacing information.

\section{CO Morphology}

We detect $\mathrm{CO}$ emission associated with the ring and a centrally peaked concentration interior to the ring (Fig. 1a). The following features are apparent. (1) The ring $\mathrm{CO}$ appears to be arranged in tightly wound spiral arms, with the azimuthally averaged peak $\mathrm{CO}$ flux about $5^{\prime \prime}$ interior to the radius of the peak $\mathrm{H} \alpha$ flux (Fig. 1b). (2) Comparison with $\mathrm{H}$ I maps of Braun (1995) at $6^{\prime \prime}$ resolution indicates that the gas interior to the ring is almost completely molecular. Near the ring, strong $\mathrm{HI}$ emission is found adjacent to $\mathrm{H}_{2}$ complexes. (3) A central molecular bar corresponds to the stellar bar. The presence of a bar and tightly wound spiral arms suggests that an OLR is present at the ring location (Yuan \& Kuo 1997), perhaps 


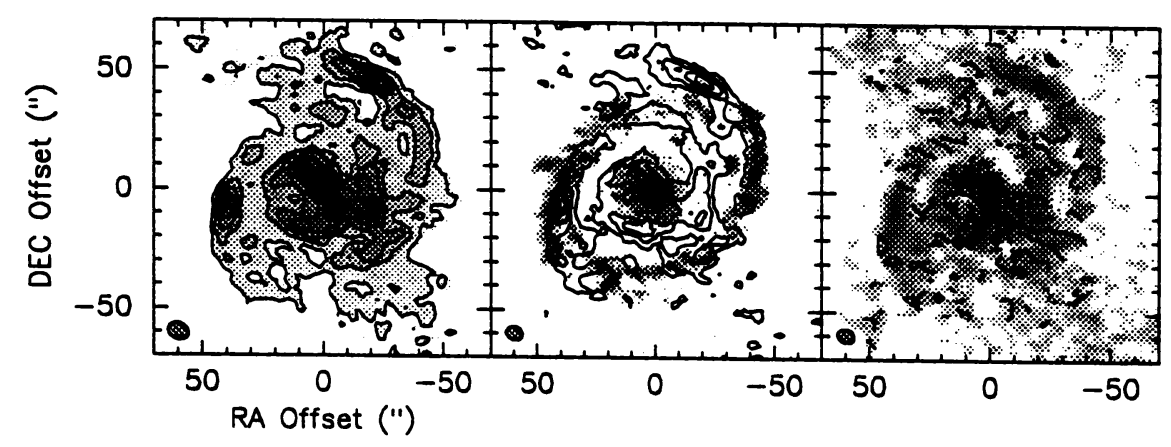

Figure 1. (a) Combined single-dish and BIMA integrated CO (1-0) map. The synthesized beam is $7^{\prime \prime} \times 9^{\prime \prime}$. (b) BIMA integrated CO map (contours) over H $\alpha$ image of González-Delgado et al. (1997). (c) BIMA velocity field, overlaid on BIMA integrated CO map. Contours are spaced by $20 \mathrm{~km} \mathrm{~s}^{-1}$.

coincident with the ILR of an oval distortion in the disk seen at $r=2^{\prime}-4^{\prime}$ (Gerin et al. 1991). (4) There is a strong one-armed spiral in the CO between the bar and the ring (Fig. 1b), and coincident with a dust lane. An $m=1$ mode is also seen on a much larger scale $\left(5^{\prime}-10^{\prime}\right)$ in H I (Braun 1995).

\section{CO Kinematics}

An isovelocity contour map derived from the BIMA data is shown in Fig. 1c, overlaid on the $\mathrm{CO}$ surface brightness image. While to first approximation the gas velocities appear circular, noncircular motions are seen associated with the central bar and the inner spiral arm. The derived rotation curve rises rapidly to its maximum within about $500 \mathrm{pc}$ of the center, indicating that the gas mass is only $\sim 5 \%$ of the dynamical mass within $r=300 \mathrm{pc}$. Subtraction of a circular rotation model yields velocity residuals suggesting inflowing gas at the ends of the central bar and, on the southern side, outflowing gas between the bar and the ring. Attempting to fit a rotation model with the same disk parameters to the $\mathrm{HI}$ data leaves a characteristic pattern of residuals indicating an increase by $\sim 7^{\circ}$ in the kinematic P.A. of the disk, a possible signature of large-scale $\mathrm{HI}$ inflow.

\section{References}

Braun, R. 1995, A\&AS, 114, 409

Burton, W.B. \& Gordon, M.A. 1978, A\&A, 63, 7

Gerin, M., Casoli, F., \& Combes, F. 1991, A\&A, 251, 32

González-Delgado, R.M. et al. 1997, ApJS, 108, 155

Möllenhoff, C., Matthias, M., \& Gerhard, O.E. 1995, A\&A, 301, 359

Sofue, Y., Honma, M., \& Arimoto, N. 1995, A\&A, 296, 33

Yuan, C. \& Kuo, C.-L. 1997, ApJ, 486, 750 